\title{
Event-Related Repetitive Transcranial Magnetic Stimulation of Posterior Superior Temporal Sulcus Improves the Detection of Threatening Postural Changes in Human Bodies
}

\author{
Matteo Candidi, ${ }^{1,2}$ Bernard M. C. Stienen, ${ }^{3}$ Salvatore Maria Aglioti, ${ }^{1,2}$ and Beatrice de Gelder ${ }^{3,4}$ \\ ${ }^{1}$ Department of Psychology, University of Rome "La Sapienza," I-00185 Rome, Italy, ${ }^{2}$ RCCS, Fondazione Santa Lucia, I-00179 Rome, Italy, ${ }^{3}$ Cognitive and \\ Affective Neuroscience Laboratory, Tilburg University, 5000 LE, Tilburg, The Netherlands, and ${ }^{4}$ Athinoula A. Martinos Center for Biomedical Imaging, \\ Massachusetts General Hospital and Harvard Medical School, Charlestown, Massachusetts 02129
}

Perceiving others' emotions through their body movements and postures is crucial for successful social interaction. While imaging studies indicate that perceiving body emotions relies upon a wide network of subcortico-cortical neural regions, little is known on the causative role of different nodes of this network. We applied event-related repetitive transcranial magnetic stimulation (rTMS) over nonfacial, body- and action-related extrastriate (EBA), temporal (pSTS), and premotor (vPM) cortices to test their active contribution in perceiving changes between two successive images of either threatening or neutral human body or animal postures. While stimulation of EBA and vPM showed no selective effect on threatening stimuli with respect to neutral ones, rTMS over pSTS selectively impaired neutral posture detection and increased the accuracy in detecting changes of threatening human postures with respect to all other experimental conditions. No such effect was found for animal stimuli. These results support the notion that pSTS is crucially devoted to the detection of socially relevant information concerning others' actions, fostering the notion that amygdalo-temporo-cortical modulatory connections mediate perception of emotionally salient body postures.

\section{Introduction}

Humans have a refined ability to use their body language to interact with others as well as with animals of other species. A specific aspect of this skill is to express one's own emotional state by means of body movements (Darwin, 1872; James, 1890). This ability is manifested by the well developed adaptive capacity to understand others' emotional state through the decoding of their body movements and postures (de Gelder, 2006; de Gelder, 2009). Its neurofunctional bases include subcortical brain regions (Hadjikhani and de Gelder, 2003; de Gelder et al., 2004; de Gelder and Hadjikani, 2006) as well as cortical sensorimotor simulative mechanisms (Rizzolatti and Craighero, 2004).

Among other nodes of the sensorimotor cortical network, the posterior superior temporal sulcus (pSTS) shows stronger activation for emotional and socially relevant body movement perception (Allison et al., 2000; Puce and Perrett, 2003; Grèzes et al., 2007; Pichon et al., 2008; de Gelder and Partan, 2009; Kret et al.,

Received Feb. 7, 2011; revised Aug. 8, 2011; accepted Oct. 3, 2011.

Author contributions: M.C., B.M.C.S., S.M.A., and B.d.G. designed research; M.C. and B.M.C.S. performed research; M.C. and B.M.C.S. analyzed data; M.C., B.M.C.S., S.M.A., and B.d.G. wrote the paper.

S.M.A. was funded by the Istituto Italiano di Tecnologia SEED (Prot. Num. 21538), the Italian Ministry of Health, and the European FP7 Grant Tango. B.d.G. was funded by The Netherlands Organisation for Scientific Research and by Tango. The project Tango acknowledges the financial support of the Future and Emerging Technologies (FET) program within the Seventh Framework Programme for Research of the European Commission, under FET-Open Grant number 249858 .

The authors declare no competing financial interests.

Correspondence should be addressed to Matteo Candidi, Department of Psychology, University of Rome "La Sapienza," Via dei Marsi 78, P.0. Box 00185, Rome, Italy. E-mail address: matteo.candidi@uniroma1.it.

DOI:10.1523/JNEUROSCI.0697-11.2011

Copyright $\odot 2011$ the authors $\quad 0270-6474 / 11 / 3117547-08 \$ 15.00 / 0$
2011), probably due to its anatomo-functional connections with the amygdalae (Amaral and Price, 1984; Morris et al., 1998; Rotshtein et al., 2001; Sah et al., 2003). Body movements, however, are not always fully visible and are often only implied in body postures.

In the last decade, researchers provided evidence for an occipito-temporal brain region specifically dedicated to the visual processing of neutral body images [extrastriate body area (EBA), Downing et al., 2001]. In particular, the right EBA is essential in discriminating morphological body details (Urgesi et al., 2004, 2007a; Moro et al., 2008), and its activity is influenced by the emotion expressed by the body (Peelen et al., 2007; Kret et al., 2011), suggesting a large-scale representation of the emotion expressed through the body.

The left vPM cortex is thought to be crucial for action representation and understanding, and is generally considered to be a key node of the "mirror neuron" system initially described in monkeys (di Pellegrino et al., 1992) consisting of bimodal visuomotor cells that fire during real action execution as well as during the passive observation of the same movement (Kilner et al., 2009). The activation of premotor regions is boosted by the emotional valence of the observed posture (de Gelder et al., 2004; Grèzes et al., 2007; Pichon et al., 2008), strengthening the notion that emotion perception is tightly linked to action programming (Darwin, 1872). Consistent with this, it has been shown that monkeys' premotor cortex receives neural projections from the amygdaloid complex (Avendaño et al., 1983) and that electrical stimulation of this area induces defensive motor behaviors (Graziano et al., 2002; Cooke and Graziano, 2004). 


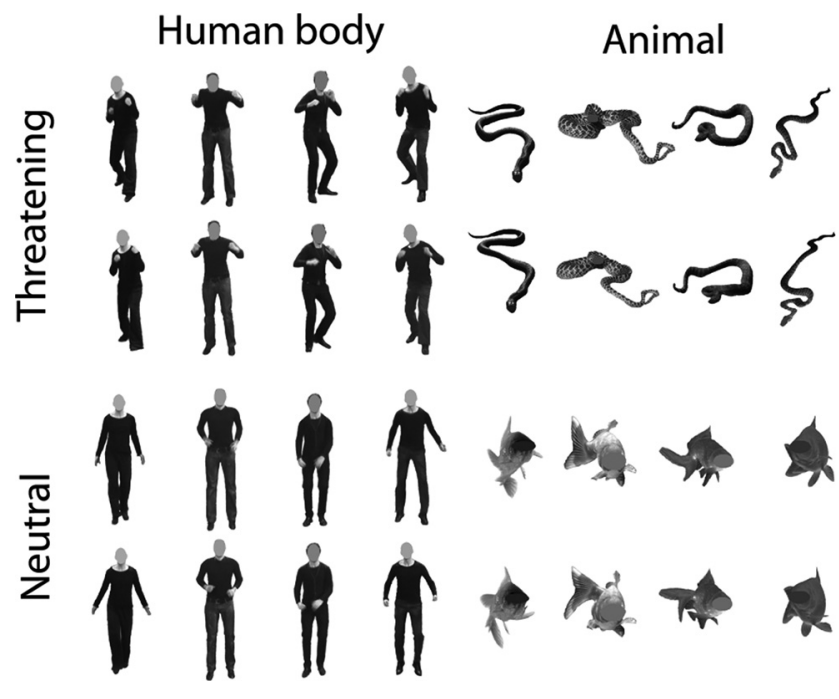

Figure 1. Complete stimulus set of threatening and neutral animal and human body images.

However, no study has thus far tested the causal contribution of right EBA, right pSTS, and left vPM cortices in detecting threatening and neutral human body postural changes. Here we used eventrelated repetitive transcranial magnetic stimulation (rTMS) to transiently alter physiological neural activity of these regions and to investigate their respective role in perceiving threatening and neutral body and animal postural changes (Kaiser et al., 2011).

\section{Materials and Methods}

Subjects. Sixteen participants (10 female) took part in the rTMS experiment (mean \pm SD age $22 \pm 1.6$ years). Thirteen participants were right handed according to the Briggs and Nebes handedness inventory (Briggs and Nebes, 1975). All participants had normal or corrected-to-normal sight. The experimental procedures were approved by the ethics committee of the Fondazione Santa Lucia (Rome, Italy) and were in accordance with the ethical standards of the 1964 Declaration of Helsinki. None of the participants had neurological, psychiatric, or other medical problems or any contraindication for rTMS (Wassermann, 1998). All subjects gave their written informed consent before the beginning of the experimental procedure, were naive to the aim of the study, and were informed about its purpose only after all experimental procedures were completed. Subjects were paid for their participation in this study.

Twelve subjects (eight female) participated in a control no-rTMS experiment (mean \pm SD age $21 \pm 1.7$ years). Ten participants were right handed according to the Briggs and Nebes handedness inventory (Briggs and Nebes, 1975). All participants had normal or corrected-to-normal sight.

Stimuli. Body images were selected from a set of dynamic video clips showing the frontal view of four male actors in black clothing jumping with their fists toward the observer (threatening body) or neutrally swinging their arms along the side of their trunk (neutral body). Two different frames were selected per actor for each condition. To test for the specificity of EBA, pSTS, and vPM cortex in the processing of human body postures, we included threatening (snakes) and harmless (fishes) animal control images. By twirling the images $\pm 30^{\circ}$ using the twirl tool in Adobe Photoshop software (Adobe Systems) and maintaining the face of the animal in the original position, two different versions of each animal were created. Both humans and animals had the face covered by a gray mask. The presentation of all stimuli was managed so as to keep the mask in the same position to preclude the possibility for the subjects to base their judgment of postural change on any change in mask position (Fig. 1).

To measure the perceived intensity of motion induced by each pair of stimuli used in the main experiment, a group of 10 independent subjects were asked to rate their subjective perception of motion during the presentation of two successive images, which showed a postural change in $50 \%$ of the trials. The timing of presentation of the stimuli and mask was

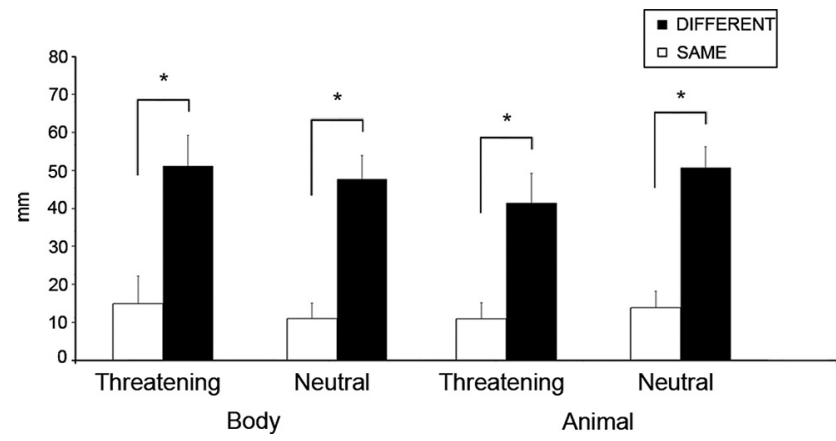

Figure 2. Subjective ratings concerning the perceived intensity of postural changes in threatening/neutral bodies and threatening/harmless animals (in millimeters; \pm SEM). $p<$ 0.05 .

the same in the behavioral and rTMS experiments. Different trials evoked higher sensation of motion with respect to same trials both in the threatening and neutral body ( $p=0.002$ and $p<0.001$ respectively) and animal ( $p=0.006$ and $p<0.001$ respectively) conditions. Moreover, as revealed by Bonferroni corrected $t$ tests against zero (the value zero represents absence of postural change perception), the perception of motion was only evoked during different trials of both threatening and neutral body and animal trials (all uncorrected $p<0.001$, while all uncorrected $p$ values $>0.01$ during same trials were non significant) (Fig. 2). Thus, only the different trials were regarded as showing the presence of the critical signal (postural change detection) for the present purposes.

On debriefing, participants were asked the following questions: (1) "What were the actors doing?" (2) "Would you define some of these postures as emotional?" and (3) "If yes, which emotion do they express?" Moreover, the participants performed a categorization task in which they had to label the seen postures as threatening or neutral. Average performance was $88 \%$ correct for recognizing the threatening postures as fighting or threatening.

Transcranial magnetic stimulation. Participants wore a tightly fitting bathing cap on which scalp stimulation points were marked. Motor evoked potentials (MEPs) were recorded from the first dorsal interosseous (FDI) muscle of the right hand. Surface $\mathrm{Ag} / \mathrm{AgCl}$ electrodes were placed in a bellytendon montage with the active electrode placed over the motor point and the reference over the interphalangeal joint. Electromyographic (EMG) signal was amplified at a gain of $1000 \times$ by a Digitimer D360 amplifier (Digitimer), bandpass filtered $(20 \mathrm{~Hz}-2.5 \mathrm{kHz})$, and digitized (sampling rate: 10 $\mathrm{kHz}$ ) by means of a CED Power 1401 controlled with Spike 2 software (Cambridge Electronic Design). The resting motor threshold (rMT), defined as the lowest intensity able to evoke 5 of 10 MEPs with an amplitude of at least $50 \mu \mathrm{V}$, was determined by holding the stimulation coil over the optimal scalp position (OSP). The OSP for inducing MEPs in the right FDI muscle was found by moving the coil in steps of $1 \mathrm{~cm}$ over the left primary motor cortex until the largest MEPs were found and then marked with a pen on a bathing cap worn by participants.

Stimulation sites were identified on each participant's scalp with SofTaxic Navigator system (EMS). Skull landmarks (nasion, inion, and two preauricular points) and $\sim 60$ points providing a uniform representation of the scalp were digitized by means of a Polaris Vicra Optical Tracking System (NDI). Coordinates in Talairach space (Talairach and Tournoux, 1988) were automatically estimated by the SofTaxic Navigator from an MRI-constructed stereotaxic template using an individualized probabilistic head model computation. This individualized head model preserves the anatomical scalp-brain correlates of a mean MR template, providing an accurate set of estimated MRI data, specific for the subject under examination. Furthermore, as the present design provided withinsite controls, no effect could be explained by errors in localizing the different sites within individuals. The scalp locations that corresponded best to right EBA, pSTS, and left vPM cortex coordinates as reported by a selected group of imaging and neurophysiological studies (Bonda et al., 1996; Decety and Grèzes, 1999; Iacoboni et al., 2001; Grossman and Blake, 2002; Nishitani et al., 2002; Pelphrey et al., 2004; Urgesi et al., 2004, 2007a,b; Michels et al., 2005; Peuskens et al., 2005; Grèzes et al., 2007; 
Table 1. Mean ( \pm SD) coordinates of the three stimulation sites according to Talairach space

\begin{tabular}{lrrr}
\hline Talairach coordinates & \multicolumn{1}{c}{ Right EBA } & \multicolumn{1}{c}{ Right pSTS } & \multicolumn{1}{c}{ Left vPM } \\
\hline$x$ & $51.7 \pm 1.1$ & $51.5 \pm 1.2$ & $-56.8 \pm 0.9$ \\
$y$ & $-71.6 \pm 1.5$ & $-47.6 \pm 0.9$ & $10.3 \pm 1.0$ \\
$z$ & $3.6 \pm 0.7$ & $9.0 \pm 0.4$ & $21.4 \pm 0.6$ \\
\hline
\end{tabular}

Candidi et al., 2008; Pichon et al., 2008; van de Riet et al., 2009) were identified and marked with a pen. Mean $( \pm S D)$ coordinates corresponded to Brodmann area 37 in the posterior part of the middle temporal gyrus (right EBA), Brodmann area 22 in the posterior part of the superior temporal sulcus (right pSTS), and to Brodmann area 44 in the pars opercularis of the inferior frontal gyrus (left vPM) (Table 1).

Repetitive TMS was performed by connecting two Magstim Model 200 stimulators with a Bistim module (The Magstim Company), producing a maximum output of $1.75 \mathrm{~T}$ at the coil surface (stimulus attenuation, $22 \%$; duration, $1 \mathrm{~ms}$; rise time, $110 \mu \mathrm{s}$ ). Two pulses were delivered with an interstimulus interval of $100 \mathrm{~ms}$ by means of a $70 \mathrm{~mm}$ figure eight stimulation coil (Magstim polyurethane-coated coil). In keeping with two previous studies showing rTMS suppressive effect on EBA during discrimination of bodyrelated features (Urgesi et al., 2004, 2007a), the first TMS pulse was delivered $150 \mathrm{~ms}$ after the onset of sample presentation. The same pulse delay was used for stimulation of pSTS and vPM cortex in keeping with magnetoencephalography and rTMS studies revealing activation of STS and ventral premotor areas within 150-200 ms after the visual presentation of moving body parts (Nishitani and Hari, 2000; Nishitani et al., 2002; Urgesi et al., 2007a,b; Candidi et al., 2008). Stimulation intensity was $120 \%$ of the rMT for both pulses and ranged from $40 \%$ to $72 \%$ (mean $=53 \%$ ) of the maximum stimulator output. During stimulation of EBA, the coil was held by hand tangential to the scalp, with the handle pointing backward and medially at a $45^{\circ}$ angle from the middle sagittal axis of the participant's head (Urgesi et al., 2004, 2007a,b; Candidi et al., 2008). During stimulation of pSTS the coil was held tangential to the scalp, with the handle pointing backward and medially at a $45^{\circ}$ angle from the middle sagittal axis of the participant's head. During stimulation of vPM cortex the coil was held tangential to the scalp, with the handle pointing backward and medially at a $45^{\circ}$ angle from the middle sagittal axis of the participant's head (Urgesi et al., 2007a,b; Candidi et al., 2008). The position of the coil with respect to the marks was checked continuously. During stimulation, participants wore commercial earplugs to protect their hearing. None of the participants reported phosphenes or hand muscular twitches after rTMS of EBA, pSTS, and vPM cortex.

Procedure. Blocks in which subjects had to detect body or animal postural changes were presented separately in alternate order. The order of stimulation site was counterbalanced within each subject according to an ABCABCCBACBA pattern. The order of the stimulated site was counterbalanced across subjects. The starting block was alternated between animals and bodies and counterbalanced across participants. A short rest was allowed before proceeding to a different block. For each task, participants completed as much practice blocks as needed to reach accuracy level above chance before proceeding to the experimental blocks. During the experimental session, two blocks of 32 trials ( 8 body neutral different, 8 body neutral same, 8 body threatening different, 8 body threatening same) were presented in each site stimulation condition, for a total of 16 trials per condition. We adopted a $2 \times 3$ (Valence $\times$ Site) factorial design.

Participants had their chin rested $57 \mathrm{~cm}$ away from a 17 inch monitor (resolution, $1151 \times 964$ pixels; refresh frequency, $60 \mathrm{~Hz}$ ), where stimuli appeared on a white background and subtended a $18 \times 9 \mathrm{~cm}$ region. Stimulus presentation timing, randomization, and rTMS triggering were controlled by E-Prime v1.1 software (Psychology Software Tools).

A trial started with the presentation of a central fixation point lasting 1000 $\mathrm{ms}$. The sample stimulus was presented for $150 \mathrm{~ms}$. After the presentation of the sample a random-dot mask $(18 \times 9 \mathrm{~cm}$ in size $)$ was presented for $200 \mathrm{~ms}$. The masks were constructed by scrambling one image from the corresponding stimulus category by custom-made software created with Matlab (The MathWorks). The custom-made software breaks down the initial image in a fixed number of dots maintaining their original grayscale. The obtained dots are randomly distributed in a space of fixed dimensions. After the disappear-

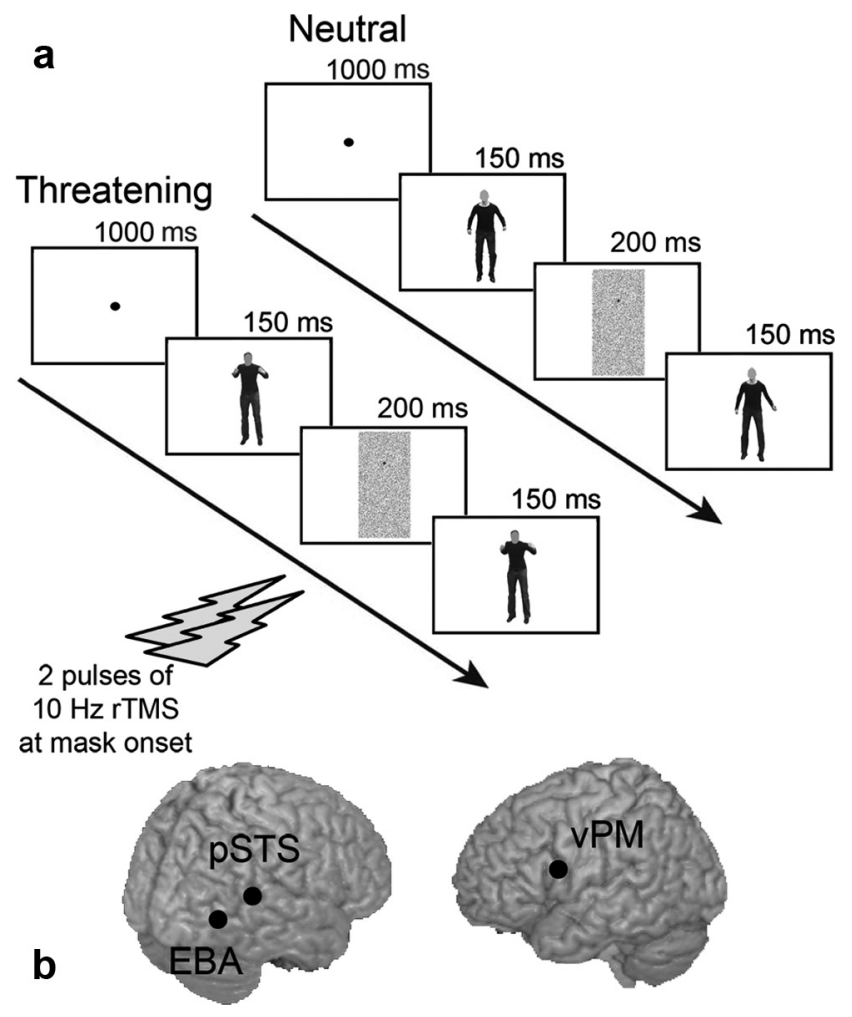

Figure 3. Timeline of the experimental procedure $(\boldsymbol{a})$ and mean coordinates of the stimulation sites $(\boldsymbol{b})$.

ance of the mask, the probe stimulus appeared on the screen for $150 \mathrm{~ms}$. A blank screen was then presented until a response was made (Fig. 3). The first pulse was delivered in coincidence with mask onset, thus $150 \mathrm{~ms}$ after sample presentation. Participants were asked to respond as quickly as possible by using their index or middle finger to press the left or the right key, respectively, on a custom-made response box. Crucially, the instruction was identical in the body and animal condition so that any differential modulation of rTMS on a given task was likely to occur at an entirely implicit level. Each key corresponded to the answer "same" or "different." The responding hand and the finger used to press the "same" and "different" button were counterbalanced across participants. Each participant was tested in a single experimental session lasting $\sim 2 \mathrm{~h}$.

Data handling. Based on the results of the subjective ratings on the perceived postural change in the different and same trials, a trial where a postural change occurred was treated as the signal. The sensitivity to the signal (detection of postural change) was estimated by calculating the $d^{\prime}$. The $d^{\prime}$ is a measure of the distance between the signal and noise distribution means in standard deviation units (Green and Swets, 1966). A $d^{\prime}$ of 0 means that the participant is not able to detect the postural change between the sample and the probe image. $d^{\prime}$ scores were calculated using the following formula:

$$
d^{\prime}=\Phi^{-1}\left(H^{\prime}\right)-\Phi^{-1}\left(\mathrm{FA}^{\prime}\right)
$$

where $\mathrm{H}^{\prime}$ is the corrected hit rate, $\mathrm{FA}^{\prime}$ is the corrected false alarm rate, and $\Phi^{-1}$ is the function that converts probabilities into $\mathrm{z}$-scores. Hit and false alarm rates were corrected for ceiling effects by applying the following formulas:

$$
\begin{gathered}
H^{\prime}=(h+0.5) /(h+m+1), \\
\mathrm{FA}^{\prime}=(f+0.5) /(f+\mathrm{cr}+1),
\end{gathered}
$$

where $h$ is the number of hits, $m$ is the number of misses, $f$ is the number of false alarms, and cr is the number of correct rejections (Snodgrass and Corwin, 1988; Tamietto et al., 2007).

Reaction times of the corresponding conditions were analyzed to control for behavioral trade-off effects. Only reaction times of correct re- 


\begin{tabular}{|c|c|c|c|c|}
\hline & EBA & pSTS & vPM & no-rTMS \\
\hline \multirow{2}{*}{\multicolumn{5}{|c|}{$\begin{array}{l}\text { Human body postural change (raw values) } \\
\qquad d^{\prime}\end{array}$}} \\
\hline & & & & \\
\hline Threatening & $2.19 \pm 0.17$ & $2.48 \pm 0.17$ & $2.09 \pm 0.13$ & $2.31 \pm 0.17$ \\
\hline Neutral & $2.31 \pm 0.13$ & $2.32 \pm 0.16$ & $2.63 \pm 0.15$ & $2.90 \pm 0.24$ \\
\hline \multicolumn{5}{|l|}{ RTs (ms) } \\
\hline Threatening & $477 \pm 32$ & $493 \pm 33$ & $485 \pm 34$ & $554 \pm 47$ \\
\hline Neutral & $482 \pm 30$ & $466 \pm 29$ & $467 \pm 29$ & $515 \pm 34$ \\
\hline \multirow{2}{*}{\multicolumn{5}{|c|}{$\begin{array}{l}\text { Animal body postural change (raw values) } \\
d^{\prime}\end{array}$}} \\
\hline & & & & \\
\hline Threatening & $2.52 \pm 0.16$ & $2.62 \pm 0.19$ & $2.57 \pm 0.14$ & $2.81 \pm 0.20$ \\
\hline Neutral & $2.96 \pm 0.13$ & $2.75 \pm 0.23$ & $3.07 \pm 0.16$ & $3.21 \pm 0.17$ \\
\hline \multicolumn{5}{|l|}{ RTs (ms) } \\
\hline Threatening & $463 \pm 29$ & $436 \pm 27$ & $474 \pm 30$ & $512 \pm 34$ \\
\hline Neutral & $442 \pm 28$ & $433 \pm 25$ & $455 \pm 26$ & $494 \pm 32$ \\
\hline
\end{tabular}

sponses were considered. Moreover, reaction times that fell below or above three standard deviations from each individual mean were identified for each condition and removed as outliers ( $0.7 \%$ of the total).

A preliminary analysis was performed on raw rTMS data (reported in Table 2). Two separate two-way repeated-measure ANOVAs with Valence (threatening/neutral) and Site (EBA/pSTS/vPM) as withinsubject factors were performed on body and animal stimuli, respectively. Analysis on raw $d^{\prime}$ in the human body posture change detection task showed that neither Site $\left(F_{(2,30)}=0.69, p=0.51\right)$ nor Valence $\left(F_{(1,15)}=1.11, p=0.31\right)$ reached statistical significance as main effects. Crucially, the interaction between Valence and Site reached statistical significance on detection of human postural change $\left(F_{(2,30)}=6.79, p=\right.$ 0.003 ). The same analysis performed on reaction times (RTs) showed a nonsignificant effect of Valence $\left(F_{(1,15)}=2.72, p=0.12\right)$, Site $\left(F_{(2,30)}=\right.$ $0.03, p=0.97)$, and their interaction $\left(F_{(2,30)}=2.24, p=0.12\right)$. The ANOVA performed on $d^{\prime}$ during the animal body postural change task revealed that detection was more difficult for threatening than neutral posture change (main effect of Valence, $F_{(1,15)}=5.53, p=0.03$ ). No effect of Site of stimulation $\left(F_{(2,30)}=0.68, p=0.51\right)$ or the interaction between Valence and Site $\left(F_{(2,30)}=1.93, p=0.16\right)$ was found. The ANOVA on RTs of the animal data showed a nonsignificant effect of Valence $\left(F_{(1,15)}=1.80, p=0.20\right)$, a significant main effect of Site $\left(F_{(2,30)}=5.47, p=0.009\right)$, which was accounted for by slower reaction times during vPM stimulation with respect to pSTS $(p=0.004)$ but not EBA $(p=0.06)$, and a nonsignificant interaction between Valence and Site $\left(F_{(2,30)}=0.73, p=0.49\right)$.

To eliminate from the analysis any task-specific difficulty difference and to be able to directly compare changes in performance during threatening and neutral detections due to rTMS, the reaction times and $d^{\prime}$ data from the rTMS experiment were transformed to $z$-scores using the control no-rTMS means and standard deviations values. The same two separated ANOVAs were performed on $z$-scores. Post hoc multiple comparisons were carried out using the Newman-Keuls test.

\section{Results}

\section{Human bodies}

The 2 Valence (threatening/neutral) $\times 3$ Site $(\mathrm{EBA} / \mathrm{pSTS} / \mathrm{vPM})$ ANOVA on $d^{\prime}$ values for human body postural change detection revealed a significant interaction between Valence and Site $\left(F_{(2,30)}=6.70, p=0.004\right)$. Crucially, post hoc tests revealed that pSTS stimulation produced a higher accuracy level during threatening $\left(d^{\prime} z\right.$-score $=0.32 \pm 0.29$ SEM $)$ compared to neutral $\left(d^{\prime}\right.$ $z$-score $=-0.71 \pm 0.19$ SEM) body postural change detection $(p<0.001)$ (Fig. 4). The dissociation between threatening and neutral stimuli was not present during stimulation of either EBA $(p=0.12)$ or $\mathrm{vPM}(p=0.81)$. Furthermore, the stimulation of pSTS during threatening body postural change detection induced higher accuracy with respect to EBA $\left(d^{\prime} z\right.$-score $=-0.19 \pm 0.29$, $p=0.02)$ and $\mathrm{vPM}\left(d^{\prime} z\right.$-score $\left.=-0.39 \pm 0.22, p=0.01\right)$ stimulation. By contrast, stimulation of the three sites did not result in any modulation of the accuracy in detecting neutral body postural changes (all $p$ values $>0.20$ ). In sum, the sensitivity of the participants to detect changes in threatening body postures was higher when stimulating pSTS than all other sites and conditions (all $p$ values $<0.02$ ). No other comparison was significant (all $p$ values $>0.09$ ). Furthermore, when compared to no-rTMS condition, only EBA and pSTS stimulation impaired performance during detection of neutral posture change (corrected $t$ tests against zero $t_{(15)}=-3.76, p=0.01$ for pSTS and $t_{(15)}=-4.63$, $p=0.002$ for EBA, all other $p>0.09$ ).

Although the Valence of the body posture did not significantly affect the $d^{\prime}$ per se $\left(F_{(1,15)}=4.27, p=0.06\right)$, detection of neutral body postural changes tended to be more difficult with respect to detection of threatening body postural changes independently from the site of stimulation. The factor Site of stimulation did not reach statistical significance as main factor $\left(F_{(2,30)}=\right.$ 0.91, $p=0.41$ ).

The ANOVA on the RTs showed no significant main effect of Valence $\left(F_{(1.15)}=0.84, p=0.37\right)$, Site $\left(F_{(2,30)}=0.06, p=0.94\right)$, or their interaction $\left(F_{(2,30)}=2.26, p=0.12\right)$. No condition resulted different from no-rTMS baseline performance (all corrected $p$ values $>0.19$ ).

\section{Animal bodies}

The 2 Valence (threatening/neutral) $\times 3$ Site $(\mathrm{EBA} / \mathrm{pSTS} / \mathrm{vPM})$ ANOVA on $d^{\prime}$ values for animal postural changes showed no significant main effect of Valence $\left(F_{(1,15)}=0.35, p=0.57\right)$, Site $\left(F_{(2,30)}=0.83, p=0.45\right)$, or their interaction $\left(F_{(2,30)}=2.06, p=\right.$ $0.15)$, thus indicating that the accuracy of the performance was not affected by rTMS over the different sites of stimulation or by the valence of animal body stimuli (Table 3 ). When compared to baseline performance, no condition resulted to be modulated (all $p$ values $>0.137)$.

The ANOVA on RTs showed a main effect of Site of stimulation $\left(F_{(2,30)}=5.31, p=0.01\right)$. Post hoc tests show that performance during pSTS stimulation was slower only with respect to $\operatorname{vPM}(p=0.008)$ regardless of the Valence of the stimuli. Neither the main effect of Valence of stimuli $\left(F_{(1,15)}=0.01, p=0.91\right)$ nor the interaction between Valence and Site of stimulation $\left(F_{(2,30)}=\right.$ $0.63, p=0.54)$ reached significance (Table 3$)$. When compared to baseline performance, no condition resulted to be modulated (all $p$ values $>0.09$ ).

\section{Discussion}

The main finding of the present research is that the right pSTS plays an opposite causal role in detecting changes in threatening and neutral human body postures. In particular, the ability to detect threatening human postural changes after pSTS stimulation was selectively modulated with respect to stimulation of body-sensitive occipitotemporal (EBA) and action-sensitive premotor ( $\mathrm{vPM}$ ) regions. These results expand previous functional imaging evidence showing the predominant involvement of pSTS in processing socially relevant bodily movements (Allison et al., 2000; Puce and Perrett, 2003; de Gelder, 2006; de Gelder and Partan, 2009; Kaiser et al., 2011) by demonstrating that this area has a crucial differential role in detecting emotional and neutral postural changes. Such an effect is probably due to the direct anatomo-functional connections between pSTS and amygdala (Amaral and Price, 1984; Morris et al., 1998; Vuilleumier et al., 2004). 


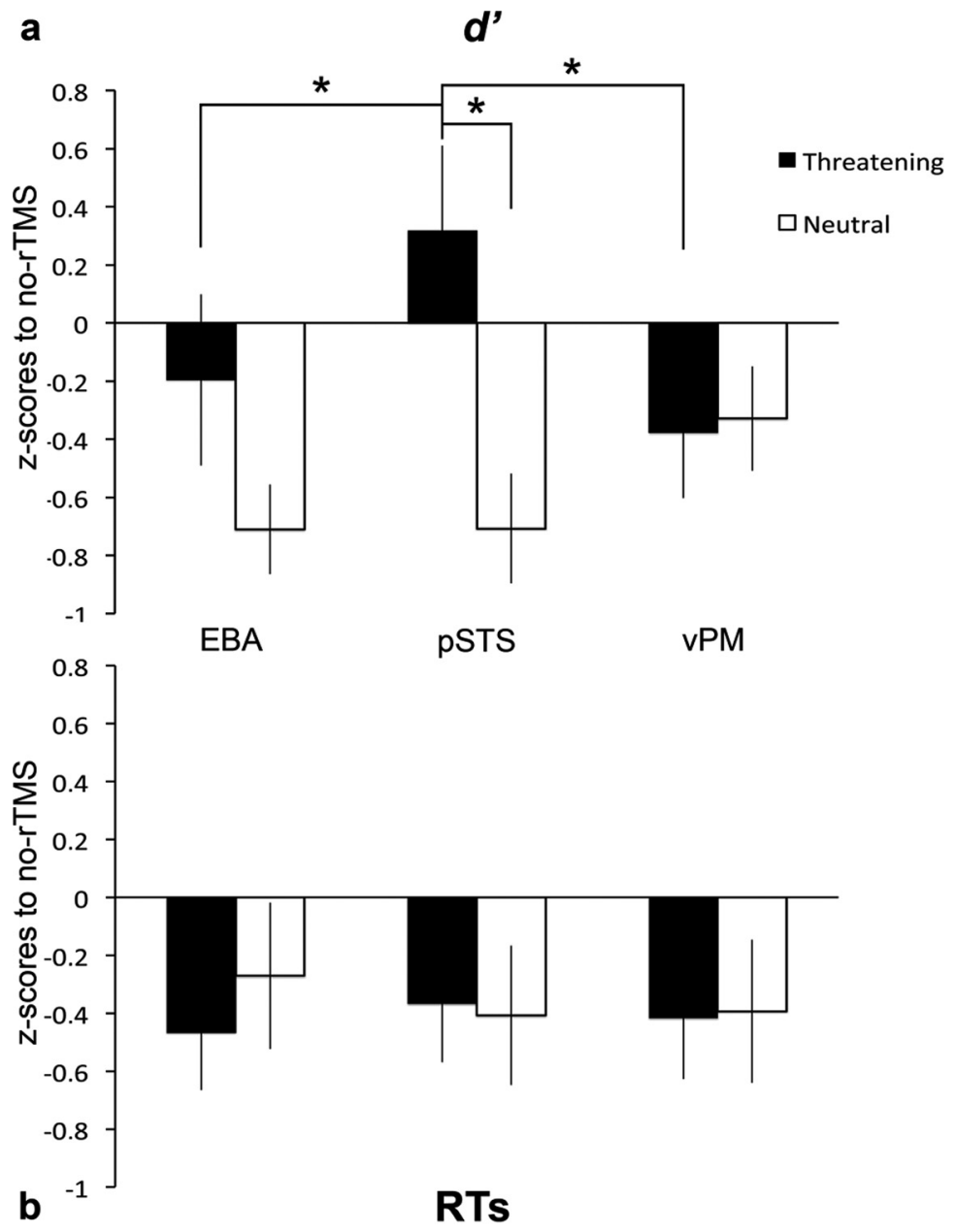

Figure 4. $\quad \boldsymbol{a}$, Accuracy $\left(d^{\prime}\right)$ in detecting threatening and neutral human body postural changes was modulated in opposite directions only during pSTS stimulation. $\boldsymbol{b}$, No effect on RTs. Error bars represent SEM. $p<0.05$.

Table 3. $z$-scores of $d^{\prime}$ and RTs (mean \pm SEM) of all experimental conditions during animal postural change detection

\begin{tabular}{llll}
\hline & EBA & pSTS & vPM \\
\hline$d^{\prime}$ & & & \\
$\quad$ Threatening & $-0.41 \pm 0.23$ & $-0.27 \pm 0.27$ & $-0.34 \pm 0.20$ \\
$\quad$ Neutral & $-0.42 \pm 0.23$ & $-0.78 \pm 0.39$ & $-0.24 \pm 0.27$ \\
RTs & & & \\
$\quad$ Threatening & $-0.41 \pm 0.24$ & $-0.63 \pm 0.23$ & $-0.31 \pm 0.25$ \\
$\quad$ Neutral & $-0.45 \pm 0.25$ & $-0.53 \pm 0.22$ & $-0.34 \pm 0.23$ \\
\hline
\end{tabular}

\section{The role of STS in threat perception}

Neurons in the posterior part of the STS respond to a variety of socially relevant stimuli such as gaze and mouth movements (Puce et al., 1998), facial expressions (Haxby et al., 2000), actions (Decety and Grèzes, 1999), biological motion (Puce and Perrett, 2003), and emotional body postures and movements (de Gelder, 2006; Kret et al., 2011; Grèzes et al., 2007; Pichon et al., 2008; de Gelder and Partan, 2009). This area contains cells whose activity is reduced when presented with pairs of successive similar body postures (Perrett et al., 2009). The quick succession of two similar images seems to induce the perception of apparent motion, thus making likely our tasks engaged brain areas involved in real motion perception. However, since in our paradigm no explicit motion was provided by the stimuli, a cautious interpretation of the results is indicated. Here we report that stimulating the pSTS has a differential effect in detecting changes between similar body postures when these convey an emotional or a neutral content. Improved accuracy in detecting threatening postural changes after right pSTS magnetic stimulation with respect to right EBA and left vPM likely occurred because of the selective sensitivity of this area to emotional (socially relevant) body postures. A variety of functional and behavioral studies support the notion that the amygdalae may strengthen the visual processing of emotional stimuli, which is thought to be carried out in extrastriate areas (Morris et al., 1998; Vuilleumier et al., 2004; Chouchourelou et al., 2006).

Although in the present experiment it is difficult to attribute the rTMS effect to perceptual or memory processes, the reported modulation is in line with previous findings (Töpper et al., 1998; Grosbras and Paus, 2003; Hayward et al., 2004; Pulvermüller et al., 2005; D'Ausilio et al., 2009). In particular, our results may expand the state-dependent TMS literature on primary and higher-order (MT/V5) visual cortices (Silvanto et al., 2007, 2008a,b; Silvanto and Muggleton, 2008; Cattaneo et al., 2008; Silvanto and Cattaneo, 2010), demonstrating that the magnetic pulse activates cells that are less active in the instant of stimulation and may thus induce both facilitatory and inhibitory perceptual effects depending on the baseline activation of the stimulated population of cells (Matthews, 1999; Siebner et al., 2009). Since cells in pSTS are more strongly activated by threatening images, TMS following the presentation of these stimuli would have activated a smaller number of cells with respect to the case of neutral stimuli (or the same number but to a lesser degree) in our study. In this condition, TMS may have induced less neural noise, increasing the signal-to-noise ratio and thus facilitating task performance with respect to when the other sites were stimulated or when neutral postural changes had to be detected. In sum, the opposite effect of rTMS over pSTS during detection of threatening and neutral posture changes may result from the interaction of the two following phenomena. First TMS activates the "less active neurons" at the precise time of stimulation and second, given pSTS connections with the amygdalae, the first visual stimulus may have activated more cells in pSTS if it was a threatening posture rather than a neutral posture. The rTMS following the first image may have thus activated fewer cells during threatening trials than during neutral ones, thus introducing less neural noise in this condition and making threatening postural change easier to be detected. This interpretation is in line with the notion that TMS over primary or higher-order (MT/V5) visual cortices during visual memory maintenance, enhances memory of specific items by reducing the signal-to-noise ratio (Cattaneo et al., 2009; Silvanto and Cattaneo, 2010). Although appealing, the statedependent interpretation of our results remains speculative. Future studies on the functional link between neural activation in pSTS and detection of emotional postural changes are needed. 
MEG and EEG studies in humans reported activation at occipitotemporal sites occurring 178-190 ms after perception of communicative mouth postures (Nishitani and Hari, 2002) and body images (Taylor et al., 2010) and showed that occipitotemporal activation is delayed for implied motion with respect to real motion perception (Lorteije et al., 2006) and for inverted compared to upright human body presentation (Stekelenburg and de Gelder, 2004). Previous rTMS and patient studies reported the crucial role of pSTS in biological motion perception (Grossman et al., 2005; Saygin, 2007), in the representation of observed actions on the basis of low-level visual features rather than highorder conceptual properties (Cattaneo et al., 2010) and in the integration between visual (mouth) and auditory (voice) information (McGurk effect) (Beauchamp et al., 2010). Moreover, delayed RTs in a gaze-shift task after STS magnetic stimulation has also been reported (Pourtois et al., 2004). Here we demonstrated, for the first time, that pSTS plays an active, crucial role in detecting emotional postural changes early in time after stimulus onset. An alternative explanation for the present results that cannot be excluded is that interference with pSTS facilitates amygdala's reaction to threatening postures, thus boosting individual's ability to detect threatening postural changes rather than neutral ones.

Beside being impaired with respect to detecting threatening human postural changes after right pSTS stimulation, detection of neutral human body postural change was also impaired with respect to baseline performance proving that our detection task causally relied on the activity of this area. This evidence is in line with studies reporting superior temporal activations during observation of static body images implying an action (Giese and Poggio, 2003; Peuskens et al., 2005) or when observing rapid succession of images in different postures (Perrett et al., 2009).

\section{EBA and vPM cortex are not selectively involved in detecting changes of emotional body postures}

Previous studies showed that the activity of EBA is crucial for local postural change detection 150-250 ms after stimulus presentation (Urgesi et al., 2007b). Although stimulation of EBA impaired detection of neutral postural change, it did not dissociate between threatening and neutral body postural changes. The involvement of EBA in responding to neutral postural changes has been shown by a previous imaging study (Downing et al., 2006). Fusiform cortex is also important for body processing (fusiform body area, Peelen and Downing, 2005) and was first reported to play a role in processing emotional bodies (Hadjikhani and de Gelder, 2003). Although EBA activity is enhanced during the presentation of emotional body postures and movements (Peelen et al., 2007; Kret et al., 2011), its activity appears not to be crucial for processing of emotional postural changes. The reported EBA activity during movement execution shown in an fMRI study (Astafiev et al., 2004) may result from late feedback signals sent from anterior motor brain regions into highlevel visual cortices.

Previous rTMS studies indicate that the vPM cortex is causatively involved in the visual discrimination of actions (Urgesi et al., 2007a; Candidi et al., 2008) and in the configural perception of body postures (Urgesi et al., 2007b). The present study shows that vPM cortex is not involved in the visual discrimination between two consecutive body postures, possibly because perception of subtle postural changes depends on higher-order visual areas (Taylor et al., 2007; Bracci et al., 2010; Cattaneo et al., 2010) rather than the sensorimotor system (Urgesi et al., 2007b). A recent TMS study provided compelling functional evidence that, while the activity of STS is involved in the visual description of observed actions (i.e., an action is linked to the body part that performs it), the activity of the left frontal gyrus is related to the representation of actions' meaning and not to the specific body part used to perform them (Cattaneo et al., 2010), supporting the notion that higher-order action-related representation are supported by premotor regions (Rizzolatti and Craighero, 2004).

\section{Conclusions}

To summarize, the present study significantly expands our understanding of the role of occipital, temporal, and premotor cortical regions in the perception of emotional and neutral body motion as seen in postural changes. The main finding is that the right pSTS plays a differential role in the processing of threatening and neutral postural changes in human bodies. This dissociation occurs at early stages of stimuli processing and is coherent with studies showing fast subcortical-cortical processing of emotional stimuli. The stimuli used in the present experiment were meant to study the role of the three stimulated areas in detecting threatening and neutral body postural changes. Thus, further research is needed to explore whether the reported effect is specific for the detection of threatening postural changes or whether it applies to emotional postural changes in general. This study shows that activity of the pSTS is not only related to the processing of socially relevant body stimuli but also crucial to effective visual detection of changing socially relevant body postures.

\section{References}

Allison T, Puce A, McCarthy G (2000) Social perception from visual cues: role of the STS region. Trends Cogn Sci 4:267-278.

Amaral DG, Price JL (1984) Amygdalo-cortical projections in the monkey (Macaca fascicularis). J Comp Neurol 230:465-496.

Astafiev SV, Stanley CM, Shulman GL, Corbetta M (2004) Extrastriate body area in human occipital cortex responds to the performance of motor actions. Nat Neurosci 7:542-548.

Avendaño C, Price JL, Amaral DG (1983) Evidence for an amygdaloid projection to premotor cortex but not to motor cortex in the monkey. Brain Res 264:111-117.

Beauchamp MS, Nath AR, Pasalar S (2010) fMRI-Guided transcranial magnetic stimulation reveals that the superior temporal sulcus is a cortical locus of the McGurk effect. J Neurosci 30:2414-2417.

Bonda E, Petrides M, Ostry D, Evans A (1996) Specific involvement of human parietal systems and the amygdala in the perception of biological motion. J Neurosci 16:3737-3744

Bracci S, Ietswaart M, Peelen MV, Cavina-Pratesi C (2010) Dissociable neural responses to hands and non-hand body parts in human left extrastriate visual cortex. J Neurophysiol 103:3389-3397.

Briggs GG, Nebes RD (1975) Patterns of hand preference in a student population. Cortex 11:230-238.

Candidi M, Urgesi C, Ionta S, Aglioti SM (2008) Virtual lesion of ventral premotor cortex impairs visual perception of biomechanically possible but not impossible actions. Soc Neurosci 3:388-400.

Cattaneo L, Sandrini M, Schwarzbach J (2010) State-dependent TMS reveals a hierarchical representation of observed acts in the temporal parietal, and premotor cortices. Cereb Cortex 20:2252-2258.

Cattaneo Z, Rota F, Vecchi T, Silvanto J (2008) Using state-dependency of transcranial magnetic stimulation (TMS) to investigate letter selectivity in the left posterior parietal cortex: a comparison of TMS-priming and TMS-adaptation paradigms. Eur J Neurosci 28:1924-1929.

Cattaneo Z, Vecchi T, Pascual-Leone A, Silvanto J (2009) Contrasting early visual cortical activation states causally involved in visual imagery and short-term memory. Eur J Neurosci 30:1393-1400.

Chouchourelou A, Matsuka T, Harber K, Shiffrar M (2006) The visual analysis of emotional actions. Soc Neurosci 1:63-74.

Cooke DF, Graziano MS (2004) Sensorimotor integration in the precentral gyrus: polysensory neurons and defensive movements. J Neurophysiol 91:1648-1660.

Darwin C (1872) The expressions of emotions in man and animals. London, UK: John Murray. Reprint. Chicago: University of Chicago Press, 1965. 
D’Ausilio A, Pulvermüller F, Salmas P, Bufalari I, Begliomini C, Fadiga L (2009) The motor somatotopy of speech perception. Curr Biol 19:381-385.

Decety J, Grèzes J (1999) Neural mechanisms subserving the perception of human actions. Trends Cogn Sci 3:172-178.

de Gelder B (2006) Towards the neurobiology of emotional body language. Nat Rev Neurosci 7:242-249.

de Gelder B (2009) Why bodies? Twelve reasons for including bodily expressions in affective neuroscience. Philos Trans R Soc Lond B Biol Sci 364:3475-3484.

de Gelder B, Hadjikhani N (2006) Non-conscious recognition of emotional body language. Neuroreport 17:583-586.

de Gelder B, Partan S (2009) The neural basis of perceiving emotional bodily expressions in monkeys. Neuroreport 20:642-646.

de Gelder B, Snyder J, Greve D, Gerard G, Hadjikhani N (2004) Fear fosters flight: a mechanism for fear contagion when perceiving emotion expressed by a whole body. Proc Natl Acad Sci U S A 101:16701-16706.

di Pellegrino G, Fadiga L, Fogassi L, Gallese V, Rizzolatti G (1992) Understanding motor events: a neurophysiological study. Exp Brain Res 91:176-180.

Downing PE, Jiang Y, Shuman M, Kanwisher N (2001) A cortical area selective for visual processing of the human body. Science 293:2470-2473.

Downing PE, Peelen MV, Wiggett AJ, Tew BD (2006) The role of the extrastriate body area in action perception. Soc Neurosci 1:52-62.

Giese MA, Poggio T (2003) Neural mechanisms for the recognition of biological movements. Nat Rev Neurosci 4:179-192.

Graziano MS, Taylor CS, Moore T (2002) Complex movements evoked by microstimulation of precentral cortex. Neuron 34:841-851.

Green DM, Swets JA (1966) Signal detection theory and psychophysics. New York: Wiley.

Grèzes J, Pichon S, de Gelder B (2007) Perceiving fear in dynamic body expressions. Neuroimage 35:959-967.

Grosbras MH, Paus T (2003) Transcranial magnetic stimulation of the human frontal eye field facilitates visual awareness. Eur J Neurosci 18:3121-3126.

Grossman ED, Blake R (2002) Brain areas active during visual perception of biological motion. Neuron 35:1167-1175.

Grossman ED, Battelli L, Pascual-Leone A (2005) Repetitive TMS over posterior STS disrupts perception of biological motion. Vision Res 45:2847-2853.

Hadjikhani N, de Gelder B (2003) Seeing fearful body expressions activates the fusiform cortex and amygdala. Curr Biol 13:2201-2205.

Haxby JV, Hoffman EA, Gobbini MI (2000) The distributed human neural system for face perception. Trends Cogn Sci 4:223-233.

Hayward G, Goodwin GM, Harmer CJ (2004) The role of the anterior cingulate cortex in the counting Stroop task. Exp. Brain Res 154:355-358.

Iacoboni M, Koski LM, Brass M, Bekkering H, Woods RP, Dubeau MC, Mazziotta JC, Rizzolatti G (2001) Reafferent copies of imitated actions in the right superior temporal cortex. Proc Natl Acad Sci U S A 98:13995-13999.

James W (1890) The principles of psychology. New York: Holt.

Kaiser MD, Shiffrar M, Pelphrey KA (2011) Socially tuned: brain responses differentiating human and animal motion. Soc Neurosci. Advance online publication. Retrieved November 15, 2011. doi:10.1082/17470919.2011. 614003.

Kilner JM, Neal A, Weiskopf N, Friston KJ, Frith CD (2009) Evidence of mirror neurons in human inferior frontal gyrus. J Neurosci 29:10153-10159.

Kret ME, Pichon S, Grèzes J, de Gelder B (2011) Similarities and differences in perceiving threat from dynamic faces and bodies. An fMRI study. Neuroimage 54:1755-1762.

Lorteije JA, Kenemans JL, Jellema T, van der Lubbe RH, de Heer F, van Wezel RJ (2006) Delayed response to animate implied motion in human motion processing areas. J Cogn Neurosci 18:158-168.

Matthews PB (1999) The effect of firing on the excitability of a model motoneurone and its implications for cortical stimulation. J Physiol 518:867-882.

Michels L, Lappe M, Vaina LM (2005) Visual areas involved in the perception of human movement from dynamic form analysis. Neuroreport 16:1037-1041.

Moro V, Urgesi C, Pernigo S, Lanteri P, Pazzaglia M, Aglioti SM (2008) The neural basis of body form and body action agnosia. Neuron 60:235-246.
Morris JS, Friston KJ, Büchel C, Frith CD, Young AW, Calder AJ, Dolan RJ (1998) A neuromodulatory role for the human amygdala in processing emotional facial expressions. Brain 121:47-57.

Nishitani N, Hari R (2000) Temporal dynamics of cortical representation for action. Proc Natl Acad Sci U S A 97:913-918.

Nishitani N, Hari R (2002) Viewing lip forms: cortical dynamics. Neuron 36:1211-1220.

Peelen MV, Downing PE (2005) Selectivity for the human body in the fusiform gyrus. J Neurophysiol 93:603-608.

Peelen MV, Atkinson AP, Andersson F, Vuilleumier P (2007) Emotional modulation of body-selective visual areas. Soc Cogn Affect Neurosci 2:274-283.

Pelphrey KA, Morris JP, McCarthy G (2004) Grasping the intentions of others: the perceived intentionality of an action influences activity in the superior temporal sulcus during social perception. J Cogn Neurosci 16:1706-1716.

Perrett DI, Xiao D, Barraclough NE, Keysers C, Oram MW (2009) Seeing the future: natural image sequences produce "anticipatory" neuronal activity and bias perceptual report. Q J Exp Psychol (Colchester) 62:2081-2104.

Peuskens H, Vanrie J, Verfaillie K, Orban GA (2005) Specificity of regions processing biological motion. Eur J Neurosci 21:2864-2875.

Pichon S, de Gelder B, Grezes J (2008) Emotional modulation of visual and motor areas by dynamic body expressions of anger. Soc Neurosci 3:199-212.

Pourtois G, Sander D, Andres M, Grandjean D, Reveret L, Olivier E, Vuilleumier P (2004) Dissociable roles of the human somatosensory and superior temporal cortices for processing social face signals. Eur J Neurosci 20:3507-3515.

Puce A, Allison T, Bentin S, Gore JC, McCarthy G (1998) Temporal cortex activation in humans viewing eye and mouth movements. J Neurosci 18:2188-2199.

Puce A, Perrett D (2003) Electrophysiology and brain imaging of biological motion. Philos Trans R Soc Lond B Biol Sci 358:435-445.

Pulvermüller F, Hauk O, Nikulin VV, Ilmoniemi RJ (2005) Functional links between motor and language systems. Eur J Neurosci 21:793-797.

Rizzolatti G, Craighero L (2004) The mirror-neuron system. Annu Rev Neurosci 27:169-192.

Rotshtein P, Malach R, Hadar U, Graif M, Hendler T (2001) Feeling or features: different sensitivity to emotion in high-order visual cortex and amygdala. Neuron 32:747-757.

Sah P, Faber ES, Lopez De Armentia M, Power J (2003) The amygdaloid complex: anatomy and physiology. Physiol Rev 83:803-834.

Saygin AP (2007) Superior temporal and premotor brain areas necessary for biological motion perception. Brain 130:2452-2461.

Siebner HR, Hartwigsen G, Kassuba T, Rothwell JC (2009) How does transcranial magnetic stimulation modify neuronal activity in the brain?Implications for studies of cognition. Cortex 45:1035-1042.

Silvanto J, Cattaneo Z (2010) Transcranial magnetic stimulation reveals the content of visual short-term memory in the visual cortex. Neuroimage 50:1683-1689.

Silvanto J, Muggleton NG (2008) Testing the validity of the TMS statedependency approach: targeting functionally distinct motion-selective neural populations in visual areas $\mathrm{V} 1 / \mathrm{V} 2$ and $\mathrm{V} 5 / \mathrm{MT}+$. Neuroimage 40:1841-1848.

Silvanto J, Muggleton NG, Cowey A, Walsh V (2007) Neural adaptation reveals state-dependent effects of transcranial magnetic stimulation. Eur J Neurosci 25:1874-1881.

Silvanto J, Muggleton N, Walsh V (2008a) State-dependency in brain stimulation studies of perception and cognition. Trends Cogn Sci 12:447-454.

Silvanto J, Cattaneo Z, Battelli L, Pascual-Leone A (2008b) Baseline cortical excitability determines whether TMS disrupts or facilitates behavior. J Neurophysiol 99:2725-2730.

Snodgrass JG, Corwin J (1988) Pragmatics of measuring recognition memory: applications to dementia and amnesia. J Exp Psychol Gen 117:34-50.

Stekelenburg JJ, de Gelder B (2004) The neural correlates of perceiving human bodies: an ERP study on the body-inversion effect. Neuroreport 15:777-780.

Talairach J, Tournoux P (1988) Co-planar stereotaxic atlas of the human brain. Stuttgart, Germany: Thieme. 
Tamietto M, Geminiani G, Genero R, de Gelder B (2007) Seeing fearful body language overcomes attentional deficits in patients with neglect. J Cogn Neurosci 19:445-454.

Taylor JC, Wiggett AJ, Downing PE (2007) Functional MRI analysis of body and body part representations in the extrastriate and fusiform body areas. J Neurophysiol 98:1626-1633.

Taylor JC, Roberts MV, Downing PE, Thierry G (2010) Functional characterisation of the extrastriate body area based on the N1 ERP component. Brain Cogn 73:153-159.

Töpper R, Mottaghy FM, Brügmann M, Noth J, Huber W (1998) Facilitation of picture naming by focal transcranial magnetic stimulation of Wernicke's area. Exp Brain Res 121:371-378.

Urgesi C, Berlucchi G, Aglioti SM (2004) Magnetic stimulation of extrastriate body area impairs visual processing of nonfacial body parts. Curr Biol 14:2130-2134.

Urgesi C, Candidi M, Ionta S, Aglioti SM (2007a) Representation of body identity and body action in extrastriate body area and ventral premotor cortex. Nat Neurosci 10:30-31.

Urgesi C, Calvo-Merino B, Haggard P, Aglioti SM (2007b) Transcranial magnetic stimulation reveals two cortical pathways for visual body processing. J Neurosci 27:8023-8030.

van de Riet WA, Grezes J, de Gelder B (2009) Specific and common brain regions involved in the perception of faces and bodies and the representation of their emotional expressions. Soc Neurosci 4:101-120.

Vuilleumier P, Richardson MP, Armony JL, Driver J, Dolan RJ (2004) Distant influences of amygdala lesion on visual cortical activation during emotional face processing. Nat Neurosci 7:1271-1278.

Wassermann EM (1998) Risk and safety of repetitive transcranial magnetic stimulation: report and suggested guidelines from the International Workshop on the Safety of Repetitive Transcranial Magnetic Stimulation, June 5-7, 1996. Electroencephalogr Clin Neurophysiol 108:1-16. 